\title{
A case manager plus psychoeducation reduced adverse outcomes in youth with type 1 diabetes
}

Svoren BM, Butler D, Levine B-S, et al. Reducing acute adverse outcomes in youths with type 1 diabetes: a randomized, controlled trial. Pediatrics 2003;112:914-22.

\section{$Q$ In children and adolescents with type 1 diabetes, does a low intensity, non-medical intervention using a case manager to monitor and encourage routine diabetes care visits, with or without supplementation by psychoeducational modules, reduce acute adverse outcomes and improve glycaemic control?}

\section{METHODS}

$\square$

Design: randomised controlled trial.

Allocation: $\{$ not concealed\}. *

Blinding: blinded \{healthcare providers and data collectors\} ${ }^{*}$

Follow up period: 24 months.

Setting: diabetes centre in Boston, Massachusetts, USA.

Patients: 301 children and adolescents aged $7-16$ years (mean age 12 y, 56\% girls, mean duration of diabetes 5.2 y) who had had type 1 diabetes for $>6$ months. Inclusion criteria included $\geqslant 1$ outpatient medical visit in the previous year, no major psychiatric problems in the patient or parent, stable living environment, and intention for routine follow up at the study centre.

$\mathbf{R}_{\mathbf{X}}$

Interventions: low intensity, non-medical intervention using a case manager (called a "Care Ambassador"), who monitored clinic attendance and assisted families with appointments (CA group, $n=95$ ); CA plus 8 sessions of psychoeducational modules over 2 years, which addressed diabetes care related issues including understanding haemoglobin $(\mathrm{Hb}) \mathrm{A}_{1 \mathrm{c}}$ concentrations, factors affecting blood sugars, team management of diabetes care, blood glucose monitoring, and carbohydrate counting (CAplus group, $\mathrm{n}=98$ ); or standard multidisciplinary diabetes care (SC group, $\mathrm{n}=108$ ).

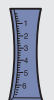

Outcomes: medical visits, hypoglycaemia, hospital admission, emergency department (ED) visits, and $\mathrm{HbA}_{1 c}$ concentrations.

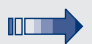

Patient follow up: $99 \%$

*Information provided by author

\section{MAIN RESULTS}

During the 24 month follow up, the CA group and the CA-plus group had a greater mean number of medical visits than the SC group (7.3 and $7.5 \vee 5.4$ visits, $\mathrm{p}=0.0001$ for the comparison of CA and CA-plus groups combined $v$ SC group); the CA and CA-plus groups did not differ $(p=0.48)$. The annual rate of severe hypoglycaemia requiring parenteral therapy was lower in the CA-plus group than in the CA and SC groups combined $(4.2 \vee 10.5$ per 100 patient $y, p=0.01)$. Also,

For correspondence: Dr L M Laffel, Joslin Diabetes Center, Harvard Medical School, Boston, MA, USA. lori.laffel@joslin.harvard.edu

Sources of funding: National Institute of Diabetes and Digestive and Kidney Diseases, Charles $\mathrm{H}$ Hood Foundation, and Katherine Adler Astrove Youth Education Fund. the annual rate of any severe hypoglycaemia was lower in the CAplus group than in the CA and SC groups combined $(45.4 v 60.6$ per 100 patient $y, p=0.02)$. The CA and CA-plus groups combined did not differ from the SC group for rates of hypoglycaemia. The annual hospital admission rate was lower in the CA-plus group than in the CA and SC groups combined ( $8.9 v 15.3$ per 100 patient $y, p=0.04)$. The annual rate of ED visits was lower in the CA-plus group than in the CA and SC groups combined $(21.0 v 34.9$ per 100 patient $y$, $\mathrm{p}=0.004)$. Mean $\mathrm{HbA}_{1 \mathrm{c}}$ concentrations did not differ among the 3 groups.

\section{CONCLUSION}

In children and adolescents with type 1 diabetes mellitus, a low intensity intervention using a case manager to monitor and encourage routine diabetes care visits, supplemented by psychoeducational modules, increased medical visits; reduced rates of severe hypoglycaemic events, hospital admission, and emergency department visits; but did not improve haemoglobin $\mathrm{A}_{1 \mathrm{c}}$ concentrations.

\section{Commentary}

Dric revious literature has shown the effectiveness of case managers in managing chronic diseases. ' However, the study by Svoren et al found that untrained care ambassadors improved adherence with clinic visits. The addition of psychoeducational modules provided by the care ambassador reduced hypoglycaemic events, severe hypoglycaemic episodes, ED visits, and hospital admissions, but had no effect on $\mathrm{HbA}_{1 \mathrm{C}}$ concentrations. Subanalysis of the high risk group showed that participants in the CA-plus group had lower mean $\mathrm{HbA}_{1 c}$ concentrations than participants in the other 2 groups combined.

The study participants attended the Joslin Diabetes Center, a world renowned diabetes centre. This may have contributed to the creation of a homogenous sample-only $7 \%$ of patients failed to meet the stringent inclusion criteria. The authors note that the intervention should be tested in a heterogeneous population. However, the care ambassador role could easily be adapted to various patient populations and clinical settings.

The results of this study are of interest to all practitioners involved in diabetes care. Clinical practice guidelines emphasise the importance of regular clinic visits for diabetes care for children. ${ }^{2}$ Care ambassadors helped to facilitate the achievement of guideline recommendations by managing non-medical aspects of care, as well as delivering written psychoeducational modules, which offered another forum for children and families to discuss diabetes related issues. The authors projected considerable savings with the introduction of care ambassadors, although no cost data were collected.

With the increasing incidence of diabetes in children ${ }^{3}$ the study by Svoren et al highlights the important role care ambassadors may have in the management of diabetes care for children and families.

Diana Sherifali, RN, BScN, CDE Cambridge Memorial Hospital Cambridge, Ontario, Canada

1 Phillips-Harris C. The integration of primary care and case management in chronic disease. Qual Manag Health Care 1996;5:1-6.

2 Canadian Diabetes Association Clinical Practice Guidelines Exper Committee. Canadian Diabetes Association 2003 clinical practice guidelines for the prevention and management of diabetes in Canada. Canadian Journal of Diabetes 2003;27:S84.

3 Schatz D, Gale EA, Atkinson MA. Why can't we prevent type 1 diabetes? Maybe it's time to try a different combination. Diabetes Care 2003:26:3326-8. 